\title{
DYNAMICS OVER TEICHMÜLLER SPACE
}

\author{
BY WILLIAM A. VEECH ${ }^{1}$
}

1. Introduction. Let $p, n \geq 0$ be such that $3 p-3+n>0$, and let $\tau_{p, n}$ be the Teichmüller space of marked closed Riemann surfaces of genus $p$ with $n$ punctures. (See $[\mathbf{1}]$ for definitions and references.) Equipped with the Teichmüller metric, $\tau_{p, n}$ is a straight line space: through each pair of distinct points there passes a unique isometric copy of $\mathbf{R}[\mathbf{3}]$. It is also known these "Teichmüller geodesics" are the projections in $\tau_{p, n}$ of orbits of a geodesic flow, the geodesic flow on the unit cotangent bundle, $\mathcal{Q}_{p, n}^{1}$, relative to a Finsler metric [9].

If $r: \mathcal{Q}_{p, n} \rightarrow \tau_{p, n}$ is the cotangent bundle projection, each $q \in \mathcal{Q}_{p, n}$ may be identified as a holomorphic quadratic differential of finite norm on any surface in the class of $r(q) \in \tau_{p, n}$. Moreover, this differential extends to be meromorphic on the closed surface with at worst simple poles at the punctures. While the choice of surface is not canonical, the singularity pattern of the differential is. That is, we may attach to $q$ a "divisor" $\sigma=\sigma(q)=(k, \nu, \varepsilon)$, where $k$ is the number of poles, $\nu(j), j \geq 1$, is the number of zeros of order $j$, and $\varepsilon=+1$ or -1 as (any representative of) $q$ is or is not the square of a 1 -form.

Given $\sigma$ as in the previous paragraph, we consider the set $\mathcal{Q}_{p, n}(\sigma)$ of $q^{\prime} \in$ $\mathcal{Q}_{p, n}$ which have $\sigma\left(q^{\prime}\right)=\sigma$. With obvious notation it is a consequence of the Teichmüller theorem that $\mathcal{Q}_{p, n}^{1}(\sigma)$ is invariant under the geodesic flow. Let $\Gamma=\Gamma(p, n)$ be the mapping class group (modular group) of a closed oriented surface of genus $p$ with $n$ punctures. $\Gamma$ acts canonically on $\mathcal{Q}_{p, n}^{1}(\sigma)$, and $\mathcal{Q}_{p, n}^{*}(\sigma)$ denotes the "moduli space", $\mathcal{Q}_{p, n}^{*}(\sigma)=\mathcal{Q}_{p, n}^{1}(\sigma) / \Gamma$. The geodesic flow commutes with $\Gamma$ and projects to a flow on $\mathcal{Q}_{p, n}^{*}(\sigma)$. We shall describe briefly the results of $[\mathbf{1 1 - 1 3}]$ : On each component of $\mathcal{Q}_{p, n}^{*}(\sigma)$ the geodesic flow is "measurably Anosov" with metric entropy a simple function of $\sigma$. A closing lemma yields a lower bound for the growth of the "Teichmüller length spectrum".

2. $F$-structures. It is possible to realize the spaces $\tau_{p, n}$ and $\mathcal{Q}_{p, n}$ as spaces of classes of atlases. To be more precise, let $M_{p, n}$ be a fixed closed oriented surface of genus $p$ with $n$ punctures, the puncture set denoted by $S_{n}=$ $\left\{s_{j} \mid 1 \leq j \leq n\right\} . H(p, n)$ (resp. $H_{0}(p, n)$ ) denotes the group of orientationpreserving homeomorphisms $\varphi$ of $M_{p}$ such that $\varphi S_{n}=S_{n}$ (resp. such that $\varphi \sim$ $\left.\operatorname{Id}\left(\operatorname{rel} S_{n}\right)\right)$. Of course, $\Gamma=H(p, n) / H_{0}(p, n)$. Let $C_{p}$ be the set of maximal

Received by the editors August 25, 1985.

1980 Mathematics Subject Classification (1985 Revision). Primary 30F99, 58F11, $58 \mathrm{~F} 15,58 \mathrm{~F} 17$.

${ }^{1}$ Research supported in part by the Institute for Advanced Study and by NSF-MCS8219148 . 
atlases of complex structures on $M_{p}$ consistent with the given orientation. If $\mathcal{U} \in \mathcal{C}_{p}$ and $\varphi \in H(p, n)$, then $\mathcal{U} \varphi=\left\{\left(\varphi^{-1} U, f \circ \varphi\right) \mid(U, f) \in \mathcal{U}\right\} \in \mathcal{C}_{p}$. The Teichmüller space is given by

$$
\tau_{p, n}=C_{p} / H_{0}(p, n) .
$$

Let $\mathbf{F}$ be the group of transformations of $\mathbf{C}$ of the form $z \rightarrow \pm z+c$. By an $F$-structure $\xi=(\mathcal{W}, N)$ on $M_{p}$ shall be understood a cofinite set $N \subseteq M_{p}$ and atlas $\mathcal{W}$ on $N$ whose transitions lie locally in $F$ and are compatible with the given orientation on $M_{p}$. It is assumed the pair $\mathcal{W}, N$ is maximal for these properties. Because the transitions belong to $F$, objects on $\mathbf{C}$ which are preserved by $F$ lift to $N$. Thus we associate to $\xi($ a) a complex structure $J(\xi)$, i.e., extension of $\mathcal{W}$ to a maximal atlas with complex analytic transitions; (b) Riemannian flat metric $g(\xi)$; (c) nowhere zero holomorphic quadratic differential $\tau(\xi)$ (pullback of $d z^{2}$ ); (d) geodesic distance function $d_{\xi}(\cdot, \cdot)$ for $g(\xi)$; and (e) volume element $V$ and norm $\|\xi\|=V(N)$. We say $\xi$ is admissible if the $d_{\xi}$-completion of $N$ is $M_{p}$. Let $\Omega(p)$ be the set of admissible $F$ structures on $M_{p}$. It may be seen that if $\xi=(\mathcal{W}, N) \in \Omega(p)$, then $J(\xi) \subseteq \mathcal{U}$ for a unique $\mathcal{U} \in \mathcal{C}_{p}$. Moreover, $\tau(\xi)$ extends to a $\mathcal{U}$-meromorphic quadratic differential on $M_{p}$ with at worst simple poles. Now we define $\Omega(p, n) \subset \Omega(p)$ to be the set of $\xi$ such that the poles of $\tau(\xi)$, if any, lie in $S_{n}(\S 1) . \Omega(p, n)$ is stabilized by $H(p, n)\left(\xi \varphi=\left(\mathcal{W} \varphi, \varphi^{-1} N\right)\right.$, and we set up the space $R_{p, n}=\Omega(p, n) / H_{0}(p, n)$. There is a canonical identity

$$
R_{p, n}=\mathcal{Q}_{p, n} .
$$

One inclusion has been indicated above; the reverse inclusion stems from associating to a quadratic differential its atlas of "natural parameters".

The Teichmüller metric is defined in terms of the quasiconformal dilatation, which in one form (circular dilatation [4]) measures how far a map is from being, infinitesimally, a complex affine motion. With $F$-structures coordinatizing domain and range (modulo a finite set), one introduces a measure, $\alpha\left(\xi_{1}, \xi_{2}\right)$, of how far Id is from being, infinitesimally, an element of $F$ [11]. In terms of $\alpha$ we define $D_{0}\left(\xi_{1}, \xi_{2}\right)=\inf \alpha\left(\xi_{1}, \xi_{2} \varphi\right), \varphi \in H_{0}(p, n)$. It is easy to see that the infimum is realized and that $D_{0}$ projects to a metric $D$ on $R_{p, n}$. One finds that $\left(R_{p, n}, D\right)$ is a complete metric space. Moreover, if $\sigma$ is a divisor as in $\S 1, R_{p, n}(\sigma)$ is an open and closed set. If $\sigma=(k, \nu, \varepsilon)$, define $N(\sigma)=2 p+k+\sum \nu(j)+(\varepsilon-3) / 2$.

2.3. THEOREM. Let $\sigma$ be such that $\mathcal{Q}_{p, n}(\sigma) \neq \varnothing . R_{p, n}(\sigma)$ admits an atlas of an $N(\sigma)$-dimensional affine complex structure with transitions which are euclidean measure preserving. With respect to the Ahlfors-Bers complex structure on $\mathcal{Q}_{p, n}, \mathcal{Q}_{p, n}(\sigma)$ is a complex analytic subvariety. The canonical map $i: \mathcal{R}_{p, n}(\sigma) \rightarrow \mathcal{Q}_{p, n}(\sigma)$ is a biholomorphism.

The theorem provides a natural everywhere positive smooth measure $\mu$ on $\mathcal{Q}_{p, n}(\sigma)$. This measure determines a measure, also denoted $\mu$, on $\mathcal{Q}_{p, n}^{1}(\sigma)$, such that $\mu$ is invariant under the geodesic flow.

2.4. THEOREM. With notations as above it is true that

$$
\mu\left(\mathcal{Q}_{p, n}^{*}(\sigma)\right)<\infty .
$$


In case $\sigma=(0,(4 p-4,0,0, \ldots),-1)$ or $\sigma=(0, \nu,+1)$ the finiteness $(2.5)$ has been proved by Masur [6]. The latter case is also a consequence of $[\mathbf{1 4}]$ and Theorem 2.3.

Let $G=\mathrm{SL}(2, \mathbf{R})$. Because $G$, as a group of linear transformations of $\mathbf{R}^{2} \cong \mathbf{C}$, normalizes the group $F, G$ acts naturally on the space $\Omega(p, n)$. One sees easily that $G H(p, n)=H(p, n) G$, and therefore the $G$ action descends to an action on $R_{p, n}$. This action preserves each stratum $R_{p, n}(\sigma)$ and each level set for the norm. Let $A$ be the diagonal subgroup, $A=\{\gamma(t)=$ $\left.\operatorname{diag}\left(e^{t / 2}, e^{-t / 2}\right) \mid t \in \mathbf{R}\right\}$, and let $N$ be the group of $2 \times 2$ upper triangular matrices with 1's on the diagonal. We remark that $\gamma(t) \sim T^{t}$ (geodesic flow) via the relation $(2.2)$.

2.6. THEOREM. Let $C$ be a topological component of $\mathcal{Q}_{p, n}^{*}(\sigma)$. The $A$ (resp. $N$ ) action is strongly mixing (resp. mixing of all orders) relative to the measure $\mu$.

In the case of the "principal stratum", $\sigma=(n,(4 p-4+n, 0,0, \ldots),-1)$, $\mathcal{Q}_{p, n}^{*}(\sigma)$ is already connected because its complement has real codimension 2 . In this case the ergodicity of the $A$ action is established in [6], and ergodicity of the $N$ action is established in [7]. In fact, once one knows, say, the $A$ action is ergodic, the existence of the ambient $G$ action implies strong mixing for both $A$ and $N$, thanks to Moore's ergodicity theorem [8]; the higher-order mixing statement for $N$ is a consequence of a general theorem by Marcus [5].

We remark that in the general case $\mathcal{Q}_{p, n}^{*}(\sigma)$ will have a finite number $\geq 2$ of components. It can be shown that already in genus three with $\sigma=$ $(0,(0,0,0,0,0,0,0,1,0, \ldots),+1)$ there are at least two components.

3. Entropy and the Teichmüller length spectrum. A somewhat more delicate analysis leads to a stronger statement about the $A$ action than is given by Theorem 2.6. $\mathcal{Q}_{p, n}^{*}(\sigma)$ is not a manifold, but it does have an open subset, with negligible (codimension 2) complement, which is a manifold. This open set, $S_{p, n}^{*}$, may be selected so as to be $A$-invariant, although it will not be $G$-invariant. We define transverse real analytic $A$-invariant dimension $N(\sigma)-1$ foliations, $\mathcal{W}^{u}$ and $\mathcal{W}^{s}$, of $S_{p, n}^{*}$. In what follows the metric $D($, on $\mathcal{Q}_{p, n}^{*}$ is the complete metric from $R_{p, n}$, transported by the correspondence (2.2).

3.1. THEOREM. Let $C$ be as in Theorem 2.6. There exists a constant $c>0$ such that for $\mu$-a.e. $x \in C$ we have

$$
\limsup _{t \rightarrow \infty} t^{-1} \log D\left(T^{t} y_{1}, T^{t} y_{2}\right) \leq-c
$$

locally uniformly in $y_{1}, y_{2} \in \mathcal{W}^{s}(x)$. A similar statement holds for $\mathcal{W}^{u}(x)$ and $t \rightarrow-\infty$; i.e., the geodesic flow is "measurably Anosov".

3.2. THEOREM. Let $C$ and $\mu$ be as in Theorem 2.6. $\left\{T^{t} \mid t \in \mathbf{R}\right\}$ is a $K$-system of entropy

$$
h_{\mu}(T)=N(\sigma) / 2 .
$$

In fact, it is possible to prove $\left\{T^{t} \mid t \in \mathbf{R}\right\}$ is Bernoulli relative to $\mu$. 
Theorem 3.2 coupled with a closing lemma yields information about the growth of the length spectrum. In what follows $\pi(C, t)$ denotes the number of periodic orbits in $C$ of least period $\leq t$; it develops that $\pi(C, t) \leq e^{b t}$ for some $b<\infty$.

3.4. THEOREM. With notations as above we have

$$
\liminf _{t \rightarrow \infty} t^{-1} \log \pi(C, t) \geq N(\sigma) / 2 \text {. }
$$

We do not know if the limit exists in (3.5) or if the right-hand side is the best lower bound. There is a bounded to one correspondence between periodic orbits for the geodesic (moduli) flow and conjugacy classes of pseudo-Anosov maps $[\mathbf{1 0}]$ in $\Gamma(p, n)$. We obtain as a corollary of Theorem 3.4 a result, a special case of which is

3.6. THEOREM. Let $\pi^{*}(p, n, t)$ be the number of primitive conjugacy classes $[\gamma] \subseteq \Gamma(p, n)$ such that there exists a pseudo-Anosov map $\varphi \in \gamma$ with expansion factor at most $e^{t}$. We have

$$
\liminf _{t \rightarrow \infty} t^{-1} \log \pi^{*}(p, n, t) \geq 6 p-6+2 n .
$$

Just as with (3.5), we do not know whether (3.7) is best possible or if the limit actually exists.

\section{REFERENCES}

1. L. Bers, Finite dimensional Teichmüller spaces and generalizations, Proc. Sympos. Pure Math., vol. 39, Amer. Math. Soc., Providence, R.I., 1983, pp. 115-156.

2. J. Hubbard and H. Masur, Quadratic differentials and foliations, Acta Math. 142 (1979), 221-274.

3. S. Kravetz, On the geometry of Teichmüller spaces and the structure of their modular groups, Ann. Acad. Sci. Fenn. Ser. A I 278 (1959), 1-35.

4. O. Lehto and K. I. Virtanen, Quasiconformal mappings in the plane, SpringerVerlag, Berlin, 1973.

5. B. Marcus, The horocycle flow is mixing of all degrees, Invent. Math. 46 (1978), 201-209.

6. H. Masur, Interval exchange transformations and measured foliations, Ann. of Math. (2) 115 (1982), 169-200.

7.

8. C. C. Moore, Ergodicity of flows on homogeneous spaces, Amer. J. Math. 88 (1966), 154-178.

9. B. O'Byrne, On Finsler geometry and application to Teichmüller spaces, Ann. of Math. Studies, No. 66, 1971, pp. 317-328.

10. W. Thurston, On the geometry and dynamics of diffeomorphisms of surfaces (preprint).

11. W. A. Veech, Dynamical systems on analytic manifolds of quadratic differentials. I, F-structures (preprint).

12. __ Dynamical systems on analytic manifolds of quadratic differentials. II, Analytic manifolds of quadratic differentials (preprint).

13. _ Dynamical systems on analytic manifolds of quadratic differentials. III, The Teichmüller geodesic flow (preprint).

14. _ Gauss measures for transformations on the space of interval exchange maps, Ann. of Math. (2) 115 (1982), 201-242.

Department of Mathematics, Rice University, Box 1892, Houston, TEXAS 77251 